\title{
Intrapersonal variation in goal setting and achievement in health coaching: cross-sectional retrospective analysis
}

\section{Opinion}

\section{Purpose of the study}

The objective of this study was to conduct a multivariate analysis of the intrapersonal differences in participants' progression in TeleHealth Coaching and goal setting, specifically "examining gender and age-related differences". ${ }^{1}$ Sample Population: The population included over 35,000 adults aged 18years or older enrolled in a HIPAA-secure International Consortium for Health and Wellness Coaching program and who set goals with their Health Coach. The majority of participants $(76 \%)$ were females, while males comprised $24 \%$ of the research study sample. The age breakdown was: 4653 of participants were younger than 30years, 18,106 were between 30 and 50years, 8663 were between 51 and 64years, and 3911 were 65years and older.

\section{Summary}

The authors' rationales for selecting this topic are the costly and preventable chronic health problems that plague the United States including obesity. The authors build a strong case for personalizing the online e-health and mobile health coaching experience to each individual for optimal health outcomes and cost savings. "Health information technologies are a critical component of our healthcare system, and often relevant to value-based care" (O'Donnell, 2018, para. 1). The key findings in this study support online health coaching, $\mathrm{m}$-Health Smartphone apps for behavior change, activity trackers and wearable as a significantly effective strategy to achieving value and cost savings in healthcare. The evidence from this research supports the use of Tele-Health Coaching for improved nutrition and fitness, tobacco cessation, weight loss and weight management, and for reducing health risks including but not limited to decreases in blood pressure, lipids, blood glucose, weight, body mass index (BMI), cholesterol, and or diabetes.

\section{Strengths of study}

The main strength of this study is the assessment of a large national population sample with demographic and operational data from a very diverse set of employers offering the same Internationally Consortium for Health and Wellness coaching program.

\section{Limitations of study} gender

This study included only two key demographic metrics, age and

\section{Recommendations for further study}

Further research should include examination of outcomes beyond goal completion or health coaching program completion. Designing a study to measure health-related outcomes, such as weight loss, BMI changes and biometric changes in health status would provide a more
Volume II Issue I - 2018

\author{
Anne MWallace, Matthew T Bogard, Susan M \\ Zbikowski, Robelyn Garcia \\ Arizona State University, USA
}

Correspondence: Robelyn Garcia, Arizona State University, Scottsdale, AZ 85260, USA, Tel 6028459802. Email DrRGarcia@asu.edu

Received: February 01, 2018 | Published: February 09, 2018

in-depth analysis of Tele-Health Coaching outcomes. Further research could also include demographic metrics of race and socioeconomic status indicators.

\section{Conclusion}

Dr. Wallace and her colleagues (2017) have provided appropriate direction and support in their efforts to develop the understanding of how successful administration of a health coaching initiative among a diverse population must be adaptable to gender and age variables and personal preference. This study revealed that women were more likely than men to choose online interactions and engage in face-toface Tele-Health Coaching sessions, whereas men were more likely to choose telephone and texting Tele-Health Coaching. Men were also more apt to engage with their health coach via mixed modalities including a combination of telephone, texting and online interactions (Figure 1). The authors also include timely content covering TeleHealth Coaching technology as a treatment modality. Integration of Tele-Health Coaching technologies have the potential to reach more patients because of the five A's of psycho technologies; accessibility, affordability, anonymity, acceptability, and adaptability (Tele Mental Health Institute [TMHI], 2018). Empirical research in Tele-Health Coaching delivered via telephone and online demonstrates it is a key intervention in health behavior change, goal setting and goal achievement. ${ }^{1-3}$ My critical review of this article is in consonance with current research and recent empirical studies affirming that Telehealth intervention can facilitate goal achievement and have a beneficial effect on the overall mental health, physical health and behavioral health of several multimorbid patient populations. ${ }^{1,4-8}$

Article published January 26, 2018 in the following e-collection issue of the Journal of Medical Internet Research:

a) Demographics of Users, Social \& Digital Divide

b) Web-based and Mobile Health Interventions

c) m-Health for Wellness, Behavior Change and Prevention 


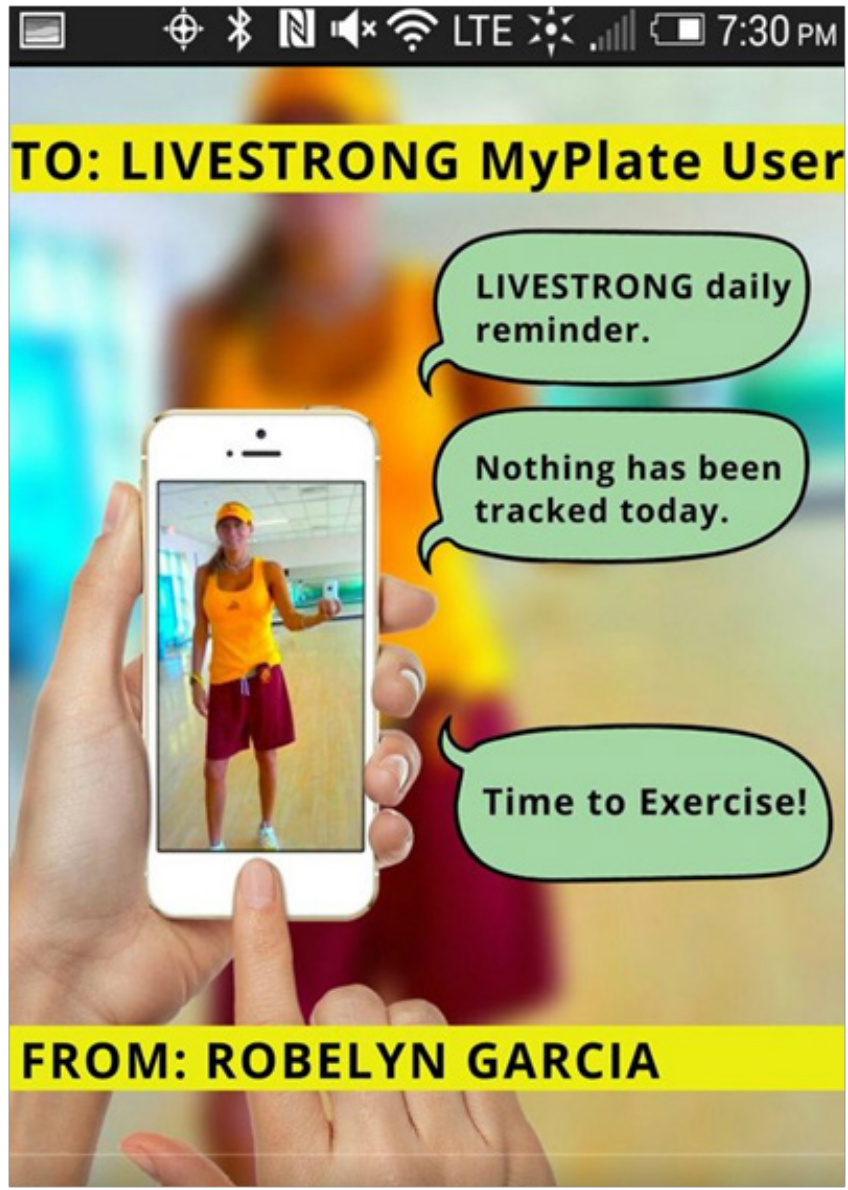

Figure I Tele-Health Coaching Text Motivation Message from Dr. Garcia via Livestrong My Plate App.

\section{Acknowledgement}

None.

\section{Conflict of interest}

The author declares no conflict of interest.

\section{References}

1. Wallace AM, Bogard MT, Zbikowski SM. Intrapersonal variation in goal setting and achievement in health coaching: Cross-sectional retrospective analysis. J Med Internet Res. 2018;20(1):e32.

2. Dejonghe LAL, Becker J, Froboese I, et al. Long-term effectiveness of health coaching in rehabilitation and prevention:A systematic review. Patient Educ Couns. 2017;100(9):1643-1653.

3. Garcia RA. Utilization, Integration \& Evaluation of LIVESTRONG's MyPlate Telehealth Technology, USA; 2015.

4. Garcia RA. Population health telehealth intervention:Medical research for treating comorbid clinical obesity and depression in geriatric patients. Part one:Review of tele-medicine scientific research. Research in Medical \& Engineering Sciences. 2017;(5):1-4.

5. Hedman E, Andersson E, jótsson B, A et al. Cost-effectiveness of Internet-based cognitive behavior therapy vs. cognitive behavioral group therapy for social anxiety disorder:Results from a randomized controlled trial. Behaviour Research and Therapy. 2011;49(11):729-736.

6. Maheu MM, Drude KP, Wright SD. Career Paths in Telemental Health. AG Switzerland: Springer International Publishing; 2017.

7. Thomas J, Bond D. Review of innovations in digital health technology to promote weight control. Current Diab Rep. 2014;14(5):1-10.

8. Does online "Working Out Work" as a treatment and prevention fordepression in older adults? An analysis of a prescribed and monitored exercise program administered via the Internet for senior adults. Arizona State University, Tempe, Arizona, USA. 